\title{
MULTIPLICITIES IN HAYMAN'S ALTERNATIVE
}

\author{
WALTER BERGWEILER and J. K. LANGLEY
}

(Received 3 September 2002; revised 28 August 2003)

Communicated by P. C. Fenton

\begin{abstract}
In 1959 Hayman proved an inequality from which it follows that if $f$ is transcendental and meromorphic in the plane then either $f$ takes every finite complex value infinitely often or each derivative $f^{(k)}, k \geq 1$, takes every finite non-zero value infinitely often. We investigate the extent to which these values may be ramified, and we establish a generalization of Hayman's inequality in which multiplicities are not taken into account.
\end{abstract}

2000 Mathematics subject classification: primary 30D35.

\section{Introduction}

Our starting point is the following result of Hayman $[8,9]$.

THEOREM 1.1. Let $k \in \mathbb{N}$ and let $a, b \in \mathbb{C}, b \neq 0$. Let $f$ be nonconstant and meromorphic in the plane. Then at least one of $f-a$ and $f^{(k)}-b$ has at least one zero, and infinitely many if $f$ is transcendental.

Hayman's proof of Theorem 1.1 is based on Nevanlinna theory, the result deduced from the following inequality. Here the notation is that of [9], with $S(r, f)$ denoting any term which is $o(T(r, f))$ as $r \rightarrow \infty$, possibly outside a set of finite Lebesgue measure.

THEOREM $1.2([8,9])$. Let $k \in \mathbb{N}$ and let $f$ be transcendental and meromorphic in the plane. Then, as $r \rightarrow \infty$,

(1) $T(r, f)<\left(2+\frac{1}{k}\right) N\left(r, \frac{1}{f}\right)+\left(2+\frac{2}{k}\right) \bar{N}\left(r, \frac{1}{f^{(k)}-1}\right)+S(r, f)$.

(C) 2005 Australian Mathematical Society $1446-7887 / 05 \$ A 2.00+0.00$ 
A normal families analogue for functions $g$ meromorphic in a plane domain $D$ was established in [7] (see also [15, 22]).

THEOREM 1.3. Let $k \in \mathbb{N}$ and let $a, b \in \mathbb{C}, b \neq 0$. Let $D$ be a plane domain, and let $F$ be the family of functions $f$ meromorphic on $D$ such that $f-a$ and $f^{(k)}-b$ have no zeros in $D$. Then $F$ is normal.

Theorems 1.1 and 1.3 together provide an illustration of the Bloch principle, that (most) properties which suffice to make constant a function meromorphic in the plane, render the corresponding family on a plane domain normal [22].

Suppose next that $f$ is meromorphic in the plane, that $n \geq 3$ and $a_{1}, \ldots, a_{n}$ are distinct elements of the extended complex plane, and that all solutions of $f(z)=a_{k}$ have multiplicity at least $m_{k} \geq 2$. A striking generalization of Picard's theorem says that if $\sum_{k=1}^{n}\left(1-1 / m_{k}\right)>2$ then $f$ is constant [9, page 46]. The investigations of the present paper started from the analogous problem involving the multiplicities of roots of $f(z)=a, f^{(k)}(z)=b$, in which direction results are not available directly from Theorem 1.2, because of the $N(r, 1 / f)$ term in (1). It will be convenient to use the following notation.

DEFINITION 1.1. Let $k$ be a positive integer and let $0<M \leq \infty, 0<N \leq \infty$. Let $D$ be a plane domain. We say that $f \in \mathfrak{F}(k, M, N, D)$ if $f$ is meromorphic on $D$ and all zeros of $f$ in $D$ have multiplicity at least $M$, while all zeros of $f^{(k)}-1$ in $D$ have multiplicity at least $N$.

Illustrating further the Bloch principle referred to above, we shall prove the following.

THEOREM 1.4. Let $k, M, N \in \mathbb{N}$ and let $D$ be a non-empty plane domain. Then $\mathfrak{F}(k, M, N, D)$ is normal if and only if $\mathfrak{F}(k, M, N, \mathbb{C})$ consists of constants only.

Theorem 1.4 will be established by applying a well-known rescaling lemma (see Lemma 5.1). In Definition 1.1 and Theorem 1.4, $M=\infty$ or $N=\infty$ should be interpreted as meaning that there are no corresponding zeros in $D$. To show that these families are not vacuous, we note that

$$
f(z)=\frac{\left(e^{z}+a\right)^{2}}{8\left(e^{z}+1\right)}, \quad a^{2}-2 a+28=0, \quad f^{\prime}(z)-1=\frac{\left(e^{z}-2\right)^{3}}{8\left(e^{z}+1\right)^{2}},
$$

gives a transcendental function $f$ in $\mathfrak{F}(1,2,3, \mathbb{C})$.

It was proved by Chen [4] that the inequality (1) holds with $N(r, 1 / f)$ replaced by $N^{(k+2)}(r, 1 / f)$, in which $N^{(k+2)}(r, 1 / f)$ is the same as $N(r, 1 / f)$ except that zeros of 
$f$ of multiplicity $k+2$ or greater are counted just $k+2$ times. It follows at once from this inequality that if

$$
\frac{(2+1 / k)(k+2)}{M_{k}}+\frac{(2+2 / k)(k+1)}{N_{k}}<1
$$

and $f \in \mathfrak{F}\left(k, M_{k}, N_{k}, \mathbb{C}\right)$ then $f$ is constant. Chen showed further in [4] that if $M_{k}, N_{k}$ satisfy (3) and $D$ is a plane domain then $\mathfrak{F}\left(k, M_{k}, N_{k}, D\right)$ is normal. We shall prove:

THEOREM 1.5. Let $k$ be a positive integer and let $0<M_{k} \leq \infty$ and $0<N_{k} \leq \infty$ with

$$
\frac{2 k+3+2 / k}{M_{k}}+\frac{(2+2 / k)(k+1)}{N_{k}}<1 .
$$

If $f \in \mathfrak{F}\left(k, M_{k}, N_{k}, \mathbb{C}\right)$ then $f$ is constant. Further, if $D$ is a plane domain then $\mathfrak{F}\left(k, M_{k}, N_{k}, D\right)$ is normal.

The condition (4) is slightly less restrictive than (3), and the normal family analogue is proved by applying a rescaling method (see Lemma 5.1), rather than the more complicated method of [4].

A generalization of Theorem 1.2 replacing the $k$ th derivative $f^{(k)}$ by a linear differential polynomial $F=f^{(k)}+\sum_{j=0}^{k-1} a_{j} f^{(j)}$ was given in [11] (see also [10]): here there are exceptional cases in which $f$ is transcendental but both $f$ and $F-1$ are zero-free. We have the following extension of the second part of Theorem 1.5.

THEOREM 1.6. Let $k, M_{k}, N_{k}, D$ be as in Theorem 1.5 , let $a_{0}, \ldots, a_{k-1}$ be functions analytic in $D$, and let $\mathfrak{G}$ be a family of functions meromorphic on $D$ with the property that, for every $f \in \mathfrak{G}$, all zeros of $f$ in $D$ have multiplicity at least $M_{k}$, and all zeros of $F-1$ have multiplicity at least $N_{k}$, in which $F=L(f)=f^{(k)}+\sum_{j=0}^{k-1} a_{j} f^{(j)}$. Then $\mathfrak{G}$ is normal.

Theorem 1.6 fails for meromorphic coefficients $a_{j}$, as the following example shows. Let

$$
f_{n}(z)=\frac{z^{j}+1}{n z}, \quad F_{n}(z)=f_{n}^{\prime}(z)+\frac{f_{n}(z)}{z}=\frac{3 z}{n}
$$

Then $F_{n}$ is non-constant on $D=B(0,1)$, and for $n \geq 3$ both $f_{n}$ and $F_{n}-1$ are zero-free there. However, the $f_{n}$ do not form a normal family. On the other hand, for functions meromorphic in the plane, we have the following, which is the key to the proof of Theorems 1.5 and 1.6. 
THEOREM 1.7. Let $f$ be meromorphic and non-constant in the plane, and let $\Lambda$ be the field of functions A meromorphic in the plane with $T(r, A)=S(r, f)$ as $r \rightarrow \infty$. Let $k$ be a positive integer, and let the linear differential operator $L_{k}$ be defined by

$$
L_{k}=D^{k}+\sum_{j=0}^{k-1} a_{j} D^{j}, \quad D=\frac{d}{d z},
$$

with the $a_{j}$ in $\Lambda$, and assume that $F=L_{k}(f)$ is non-constant. If $L_{k} \neq D^{k}$ set

$$
Q=\max \left\{j: 0 \leq j \leq k-1, a_{j} \not \equiv 0\right\}, \quad q=\max \{1, k-1-Q\},
$$

while if $L_{k}=D^{k}$ set $Q=-1, q=k$. Then one of the following holds.

(i) We have

$$
\begin{aligned}
T(r, f)< & 2 N^{(k+1)}\left(r, \frac{1}{f}\right)+\frac{1}{q} N^{(k+2)}\left(r, \frac{1}{f}\right) \\
& +\left(2+\frac{2}{q}\right) \bar{N}\left(r, \frac{1}{F-1}\right)+S(r, f),
\end{aligned}
$$

in which $N^{(p)}(r, 1 / f)$, for $p \in \mathbb{N}$, counts zeros of $f$, but with zeros of multiplicity at least $p$ counted just $p$ times.

(ii) There exists a function $H$ meromorphic in the plane and satisfying

$$
\beta(z)=\frac{H^{\prime \prime}(z)}{H^{\prime}(z)}=\frac{2 a_{k-1}(z)}{k(k+1)},
$$

such that $f, L_{k}$ and $F$ are given by

$$
\begin{gathered}
f=\frac{(H-\omega)^{k+1}}{k ! H\left(H^{\prime}\right)^{k}}, \quad L_{k}=(D+\beta) \cdots(D+k \beta), \\
F=L_{k}(f)=1-1 / H^{k+1},
\end{gathered}
$$

in which $\omega$ is $a(k+1)$ th root of unity.

If $a_{k-1} \equiv 0$, then (i) always holds.

It is clear that by choosing $\beta, H, f, L_{k}$ as in (8) and (9) it is possible to ensure that $f$ is transcendental but $f$ and $F-1$ are zero-free. Theorem 1.7 contains Theorem 1.2 and the results of $[4,10,11]$, and the proof presented here is considerably simpler than that in [11]. It seems extremely unlikely, however, that the constants in (4) and (7) are sharp, and it seems reasonable to investigate the extent to which the condition on one of $M_{k}, N_{k}$ may be relaxed, if a stronger condition is placed on the other. In this direction, we have the following result. 
THEOREM 1.8. Let $k$ be a positive integer. Then there exists a positive integer $T_{k}$ with the following property. If $D$ is a plane domain then the family $\mathfrak{F}\left(k, k+2, T_{k}, D\right)$ is normal, and if $f \in \mathfrak{F}\left(k, k+2, T_{k}, \mathbb{C}\right)$ then $f$ is constant.

We remark that, with $k$ a positive integer, Wang and Fang proved in [17, Theorem 7] that $\mathfrak{F}(k, k+2, \infty, D)$ is normal for every plane domain $D$, and [17, Theorem 3] that $\mathfrak{F}(k, 3, \infty, \mathbb{C})$ contains only rational functions, the simple example $f(z)=z^{3}$ showing that $\mathfrak{F}(k, 3, \infty, \mathbb{C})$ does have non-constant elements.

The proof of Theorem 1.8 uses the rescaling lemma and the following two propositions due to Wang and Fang [17, Lemmas 8 and 6].

PROPOSITION 1.9 ([17]). Let $k$ be a positive integer. Then $f$ is a non-constant rational function in $\mathfrak{F}(k, k+1, \infty, \mathbb{C})$ if and only if $f$ has the form

$$
f(z)=\frac{(z+a)^{k+1}}{k !(z+b)}, \quad a, b \in \mathbb{C}, \quad a \neq b .
$$

The examples (10) show that the family $\mathfrak{F}(k, k+1, \infty, D)$ is not normal for a plane domain $D$, since $a$ may be taken arbitrarily close to $b$.

PROPOSITION 1.10 ([17]). Let $k \in \mathbb{N}$ and let $f$ be transcendental and meromorphic of finite order in the plane, such that $f^{(k)}-1$ has finitely many zeros. Then $f$ has infinitely many zeros of order $k$ or less.

In particular Proposition 1.10 shows that $\mathfrak{F}(k, k+1, \infty, \mathbb{C})$ contains no transcendental function of finite order. It is not clear whether Proposition 1.10 holds without the hypothesis that $f$ has finite order, the proof in [17] relying on a result from [2] concerning critical and asymptotic values. Indeed, it was conjectured in [1] that if $f$ is transcendental and meromorphic in the plane with $f^{\prime}-1$ zero-free then there exists a sequence $z_{n} \rightarrow \infty$ with $f\left(z_{n}\right)=0$ and $f^{\prime}\left(z_{n}\right) \rightarrow \infty$. This is known to be true if in addition $f$ has finite order [1, Lemma 5] or $f$ has at most finitely many multiple poles [12, Theorem 2].

For rational functions we prove the following:

THEOREM 1.11. Let $k \in \mathbb{N}, M \geq k+1$ and $N \geq 2$. Let $f$ be a non-constant rational function in $\mathfrak{F}(k, M, N, \mathbb{C})$. Then

$$
\frac{k}{M}+\frac{k+1}{N}>1
$$

or $f$ has the form (10). 
Theorem 1.11 is proved via complex dynamics, using ideas introduced to the area by Eremenko [6]. Taking $N=\infty$ shows that Theorem 1.11 contains Proposition 1.9. We give two examples of non-constant rational functions in $\mathfrak{F}(1, M, N, \mathbb{C})$ satisfying (11) with $k=1$ and $M, N \geq 2$.

EXAMPLE 1.1. Let $N=2, M \geq 3$ and

with $c \in \mathbb{C} \backslash\{0\}$. Then

$$
f(z)=\frac{4(M-1)}{M^{2}} \frac{z^{M}}{z^{M-1}+c}
$$

$$
f^{\prime}(z)-1=-\frac{\left(M c-(M-2) z^{M-1}\right)^{2}}{M^{2}\left(z^{M-1}+c\right)^{2}} .
$$

EXAMPLE 1.2. Let $N=3, M=2$ and

$$
f(z)=\frac{(7+i)\left(z^{2}-1\right)^{2}}{8 z\left(z^{2}-1+2 i\right)}, \quad f^{\prime}(z)-1=\frac{(i-1)\left(z^{2}+1-2 i\right)^{3}}{8 z^{2}\left(z^{2}-1+2 i\right)^{2}} .
$$

On the other hand, it seems unlikely that Theorem 1.11 is sharp for $k \geq 2$. To show that Theorem 1.11 fails for transcendental meromorphic functions, we give examples of transcendental functions $f$ in $\mathfrak{F}(1, M, N, \mathbb{C})$, for which equality holds in (11) with $k=1$.

EXAMPLE 1.3. The function

$$
f(z)=\left(e^{-4 z}+1\right)^{-1}, \quad f^{\prime}(z)-1=-\left(e^{-4 z}-1\right)^{2}\left(e^{-4 z}+1\right)^{-2},
$$

has $M=\infty, N=2$.

Next, $f(z)=z-\tan z$ is an obvious example for $(M, N)=(1, \infty)$, and any elliptic solution $f$ of $\left(f^{\prime}\right)^{2}=1-(f+1)^{4}$ provides an example for $(2,4)$.

EXAMPLE 1.4. Let $f=1 / \wp^{\prime}$, where $\wp$ is the Weierstraß doubly periodic function satisfying $\left(\wp^{\prime}\right)^{2}=4 \wp^{3}-g_{2} \wp-g_{3}=4 \wp^{3}+3 \wp-1$. Then $f$ has only triple zeros and so has $f^{\prime}-1=-4(\wp+1 / 2)^{3} /\left(\wp^{\prime}\right)^{2}$, so that $f \in \mathfrak{F}(1,3,3, \mathbb{C})$.

EXAMPLE 1.5. Let $f(z)=1 /\left(a \tan ^{3} z+b \tan z\right)$. For suitable values $a, b, u, v$ we then have $f^{\prime}(z)-1+f(z)^{2}\left(u \tan ^{2} z+v\right)^{3}=0$, and again $f$ and $f^{\prime}-1$ have only zeros of multiplicity at least 3 , so that $f \in \mathfrak{F}(1,3,3, \mathbb{C})$.

A specific solution of the equations for $a, b, u, v$ (found with MAPLE) is, with $x=\sqrt[1]{2}$

$$
\begin{array}{ll}
a=\frac{3-24 x+17 x^{2}}{25}, & u=\frac{\left(75+150 x-75 x^{2}\right)^{1 / 3}\left(-11+8 x+x^{2}\right)}{75}, \\
b=\frac{-3-6 x+3 x^{2}}{5}, & v=-\frac{\left(75+150 x-75 x^{2}\right)^{1 / 3}}{5} .
\end{array}
$$


EXAMPLE 1.6. Let

$$
f(z)=\frac{1}{\frac{1}{4} \sqrt{3} \tan ^{2} z-\frac{1}{2} \tan z+\frac{5}{12} \sqrt{3}}
$$

so that

$$
f^{\prime}(z)-1=-\frac{f(z)^{2}}{48}(\sqrt{3} \tan z+1)^{4} .
$$

Then $f \in \mathfrak{F}(1,2,4, \mathbb{C})$.

A (bold) conjecture might be that if $f$ is a non-constant function in $\mathfrak{F}(k, M, N, \mathbb{C}$ ) then $k / M+(k+1) / k N \geq 1$, possibly with strict inequality if $f$ is a rational function. For $k=2$, the following examples would then be extremal.

EXAMPLE 1.7. Let $\wp$ again denote the Weierstraß doubly periodic function, this time with $g_{2}=-4 / 5, g_{3}=-24 / 5$, and set $f(z)=(\wp(z)+1)^{-2}$. Then

$$
f^{\prime \prime}(z)-1=-(\wp(z)-3)^{3} /(\wp(z)+1)^{3}
$$

so that $f \in \mathfrak{F}(2,4,3, \mathbb{C})$.

EXAMPLE 1.8. Let $g_{2}=0, g_{3}=196 / 5$ and $f(z)=\wp(z)^{-4}$. Then

$$
f^{\prime \prime}(z)-1=-\left(\wp(z)^{3}-28\right)^{2} / \wp(z)^{6}
$$

so that $f \in \mathfrak{F}(2,8,2, \mathbb{C})$.

We mention some further related results. First, a modified version of (1) was proved by Yang Le in [18], but it is not clear whether those methods can be adapted to give an inequality on the lines of (7). Next, the questions considered in this paper were addressed by Yang and Zhang [19] for holomorphic functions. It is shown in [19] that for a plane domain $D$ and a positive integer $k$, the family of holomorphic functions in $\mathfrak{F}\left(k, M_{k}, N_{k}, D\right)$ is normal if $(k+1) / M_{k}+1 / N_{k}<1$. Results for meromorphic functions were obtained in [20], but subject to additional hypotheses on the multiplicities of poles. A survey of some results may be found in [5]. Finally, further results on normal families of meromorphic functions with multiple zeros and poles and $f^{\prime}-1$ zero-free appear in [17].

\section{Proof of Theorem 1.7}

Let $f$ and $F$ be as in the hypotheses. We start with Milloux' inequality [9, page 57]

(13) $T(r, f)<\bar{N}(r, f)+N\left(r, \frac{1}{f}\right)+\bar{N}\left(r, \frac{1}{F-1}\right)-N_{0}\left(r, \frac{1}{F^{\prime}}\right)+S(r, f)$, 
in which $N_{0}\left(r, 1 / F^{\prime}\right)$ counts only zeros of $F^{\prime}$ which are not zeros of $F-1$. Let $N^{*}\left(r, 1 / F^{\prime}\right)$ count zeros of $F^{\prime}$ which are not zeros of $F-1$ and, in addition, are not zeros of $f$ of multiplicity $k+2$ or greater.

\section{LEMMA 2.1. We have}

$$
N\left(r, \frac{1}{f}\right)+N^{*}\left(r, \frac{1}{F^{\prime}}\right) \leq N^{(k+1)}\left(r, \frac{1}{f}\right)+N_{0}\left(r, \frac{1}{F^{\prime}}\right)+S(r, f) .
$$

PRoof. Suppose that at least one of $f$ and $F^{\prime}$ has a zero at $a$. If $f$ has a zero at $a$ denote its multiplicity by $m$, and set $m=0$ if $f(a) \neq 0$. Suppose first that $m \leq k+1$. Then $a$ contributes the same to $n^{*}\left(r, 1 / F^{\prime}\right)$ as to $n_{0}\left(r, 1 / F^{\prime}\right)$, and contributes $m$ to each of $n(r, 1 / f)$ and $n^{(k+1)}(r, 1 / f)$.

Assume next that $a$ is a zero of $f$ of multiplicity $m \geq k+2$, and write $1 / f=$ $(F / f)(1 / F)$. Let $p_{j} \geq 0$ be the order of the pole of $a_{j}$ at $a$, with $p_{j}=0$ if $a_{j}(a)$ is finite. Similarly, let $\mu$ be the order of the zero of $F$ at $a$, with $\mu=0$ if $F(a) \neq 0$. Since each $f^{(j)} / f$ has poles of multiplicity at most $j$ and $F / f=f^{(k)} / f+\sum_{j=0}^{k-1} a_{j} f^{(j)} / f$, we get

$$
m \leq k+\mu+\sum_{j=0}^{k-1} p_{j}=k+1+\mu-1+\sum_{j=0}^{k-1} p_{j} .
$$

Now $a$ contributes exactly $m$ to $n(r, 1 / f)+n^{*}\left(r, 1 / F^{\prime}\right)$. On the other hand using (15) we see that, whether or not $\mu$ is positive, $a$ contributes at least

$$
(k+1)+(\mu-1) \geq m-\sum_{j=0}^{k-1} p_{j}
$$

to $n^{(k+1)}(r, 1 / f)+n_{0}\left(r, 1 / F^{\prime}\right)$, and this proves the lemma.

Using Lemma 2.1, inequality (13) now becomes

$$
\begin{aligned}
T(r, f)< & \bar{N}(r, f)+N^{(k+1)}\left(r, \frac{1}{f}\right)+\bar{N}\left(r, \frac{1}{F-1}\right) \\
& -N^{*}\left(r, \frac{1}{F^{\prime}}\right)+S(r, f) .
\end{aligned}
$$

Following Hayman [9, page 60], write

$$
\bar{N}(r, f)=N_{1}(r, f)+\bar{N}_{2}(r, f),
$$

in which $N_{1}(r, f)$ counts simple poles of $f$, while $\bar{N}_{2}(r, f)$ counts the points at which $f$ has multiple poles, each such pole counted just once. Since

$$
N_{1}(r, f)+2 \bar{N}_{2}(r, f) \leq N(r, f) \leq T(r, f),
$$


we obtain from (16) and (17),

$$
\bar{N}_{2}(r, f)<N^{(k+1)}\left(r, \frac{1}{f}\right)+\bar{N}\left(r, \frac{1}{F-1}\right)-N^{*}\left(r, \frac{1}{F^{\prime}}\right)+S(r, f) .
$$

We may therefore assume henceforth that

$$
N_{1}(r, f) \neq S(r, f) \text {, }
$$

because if (19) fails, then using (17) and (18) in (16) leads at once to an inequality stronger than (7).

\section{LEMMA 2.2. Let}

$$
M(z)=(k+1) \frac{F^{\prime \prime}(z)}{F^{\prime}(z)}-(k+2) \frac{F^{\prime}(z)}{F(z)-1}-\frac{2 a_{k-1}(z)}{k} .
$$

Assume that $f$ has a simple pole at $b$, and that $b$ is not a pole of any of the coefficients $a_{j}$. Then $M$ has a zero of multiplicity at least $q=\max \{1, k-1-Q\}$ at $b$, in which $Q$ is defined as in Theorem 1.7.

PROOF. Assume first that $Q=k-1$, so that $q=1$. As $z \rightarrow b$ we may write

$$
f(z)=d(z-b)^{-1}+O(1)
$$

with $d$ a non-zero complex number,

$$
\begin{aligned}
F(z)-1= & (-1)^{k} k ! d(z-b)^{-k-1}+ \\
& +a_{k-1}(b)(-1)^{k-1}(k-1) ! d(z-b)^{-k}+O(|z-b|)^{-k+1}
\end{aligned}
$$

and

$$
\begin{aligned}
F^{\prime}(z)= & (-1)^{k+1}(k+1) ! d(z-b)^{-k-2}+ \\
& +a_{k-1}(b)(-1)^{k} k ! d(z-b)^{-k-1}+O(|z-b|)^{-k}
\end{aligned}
$$

This gives, as $z \rightarrow b$,

and

$$
\frac{F^{\prime}(z)}{F(z)-1}=-\frac{k+1}{z-b}-\frac{a_{k-1}(b)}{k}+O(|z-b|)
$$

$$
\frac{F^{\prime \prime}(z)}{F^{\prime}(z)}=-\frac{k+2}{z-b}-\frac{a_{k-1}(b)}{k+1}+O(|z-b|),
$$

and we see at once that $M(b)=0$. 
Next, suppose that $0 \leq Q<k-1$, so that in particular $a_{k-1} \equiv 0$ and $q=k-1-Q$. Then as $z \rightarrow b$ we have

$$
\begin{aligned}
F(z)-1 & =(-1)^{k} k ! d(z-b)^{-k-1}+O(|z-b|)^{-Q-1} \\
F^{\prime}(z) & =(-1)^{k+1}(k+1) ! d(z-b)^{-k-2}+O(|z-b|)^{-Q-2}
\end{aligned}
$$

and

$$
\frac{F^{\prime}(z)}{F(z)-1}=-\frac{k+1}{z-b}+O(|z-b|)^{q}, \quad \frac{F^{\prime \prime}(z)}{F^{\prime}(z)}=-\frac{k+2}{z-b}+O(|z-b|)^{q},
$$

so that $M$ has a zero of multiplicity at least $q$ at $b$. Finally, suppose that $Q=-1$, so that $q=k$. Then as $z \rightarrow b$ we have

$$
\begin{aligned}
F(z)-1 & =(-1)^{k} k ! d(z-b)^{-k-1}+O(1), \\
F^{\prime}(z) & =(-1)^{k+1}(k+1) ! d(z-b)^{-k-2}+O(1),
\end{aligned}
$$

which gives (21) again.

\section{Completion of the proof of Theorem 1.7, when $M \not \equiv 0$}

Suppose that $M \not \equiv 0$ in (20). Then by Lemma 2.2 we have

$$
q N_{1}(r, f) \leq N\left(r, \frac{1}{M}\right)+S(r, f) \leq T(r, M)+S(r, f) \leq N(r, M)+S(r, f) .
$$

But poles of $M+2 a_{k-1} / k$ can only be simple, and can only arise from poles of the $a_{j}$, multiple poles of $f$, zeros of $F-1$, zeros of $F^{\prime}$ which contribute to $N^{*}\left(r, 1 / F^{\prime}\right)$, and zeros of $f$ of multiplicity at least $k+2$. Since a zero of $f$ of multiplicity at least $k+2$ contributes 1 to $n^{(k+2)}(r, 1 / f)-n^{(k+1)}(r, 1 / f)$, this gives

$$
\begin{aligned}
q N_{1}(r, f)< & \bar{N}_{2}(r, f)+\bar{N}\left(r, \frac{1}{F-1}\right)+N^{*}\left(r, \frac{1}{F^{\prime}}\right)+ \\
& +N^{(k+2)}\left(r, \frac{1}{f}\right)-N^{(k+1)}\left(r, \frac{1}{f}\right)+S(r, f),
\end{aligned}
$$

which, together with (18), leads to

$$
q N_{1}(r, f)<N^{(k+2)}\left(r, \frac{1}{f}\right)+2 \bar{N}\left(r, \frac{1}{F-1}\right)+S(r, f) .
$$

Using (17), (18) and (22) in (16), we obtain (7). 


\section{Completion of the proof of Theorem 1.7 , when $M \equiv 0$}

Assume henceforth that $M \equiv 0$ in (20), and define the function $\beta$ by

$$
\beta(z)=\frac{2 a_{k-1}(z)}{k(k+1)} .
$$

Since we are assuming that (19) holds, we may take a simple pole $z_{1}$ of $f$ and a small simply connected neighbourhood $U$ of $z_{1}$ on which all of the coefficients $a_{0}, \ldots, a_{k-1}$ are analytic. It follows therefore that we may define an analytic branch $H$ of $(1-F)^{-1 /(k+1)}$ on $U$, with a simple zero at $z_{1}$. Computation of $H^{\prime}$ and $H^{\prime \prime} / H^{\prime}$ then shows that $H$ is a solution of (8) in $U$, since $M \equiv 0$ in (20), and obviously we have

$$
F=L_{k}(f)=1-1 / H^{k+1}
$$

Define the linear differential operators $J, K$ by

$$
J=(D+\beta) \cdots(D+k \beta), \quad K=L_{k}-J, \quad D=\frac{d}{d z},
$$

and define a function $N$, meromorphic on $U$, by

$$
N=\frac{H^{k+1}-(-1)^{k}}{k ! H\left(H^{\prime}\right)^{k}} .
$$

Then (8) gives

$$
(D+k \beta)(N)=\frac{1}{\left(H^{\prime}\right)^{k-1}} \frac{d}{d H}\left(\frac{H^{k+1}-(-1)^{k}}{k ! H}\right)
$$

and repeating this shows that, on $U$,

$$
J(N)=1-1 / H^{k+1}=F .
$$

LEMMA 4.1. The linear differential operator $K$ is the zero operator.

PROOF. Suppose that $K$ is not the zero operator. Since the coefficients of $K$ are in the field $\Lambda$, and since (19) holds, we may assume that $z_{1}$ has been chosen so that $K(f)$ has a pole at $z_{1}$. Write

$$
f(z)=\frac{c}{z-z_{1}}+O(1), \quad H(z)=d\left(z-z_{1}\right)+O\left(\left|z-z_{1}\right|\right)^{2},
$$

as $z \rightarrow z_{1}$. Then a comparison of Laurent series in (26) gives

$$
-\frac{1}{d^{k+1}}=(-1)^{k} k ! c, \quad c=\frac{(-1)^{k+1}}{k ! d^{k+1}} .
$$


On the other hand,

$$
N(z)=\frac{(-1)^{k+1}}{k ! d^{k+1}\left(z-z_{1}\right)}+O(1)=\frac{c}{z-z_{1}}+O(1)
$$

as $z \rightarrow z_{1}$, and so $N-f$ is analytic at $z_{1}$. But (24) and (26) give

$$
K(f)=L_{k}(f)-J(f)=F-J(f)=J(N-f),
$$

and this is a contradiction, since $K(f)$ has a pole at $z_{1}$, by assumption, but $J(N-f)$ is regular there.

LEMMA 4.2. Either (7) holds, or $H$ is meromorphic in the plane.

Proof. Let $V=H^{k+1}, w=(D+2 \beta) \cdots(D+k \beta) f$, with $w=f$ if $k=1$. Then (23) gives $(D+\beta) w=F=1-1 / H^{k+1}=1-1 / V$, from which we see that $V$ is meromorphic in the plane. Integration gives, on $U$,

$$
w=\frac{(k+1)(k V+1)}{k V^{\prime}}+\frac{c}{H^{\prime}},
$$

in which $c$ is a constant. If $c \neq 0$ then $H^{\prime}$ is meromorphic in the plane, and so is $H$, since $V^{\prime} / V=(k+1) H^{\prime} / H$. Suppose next that $c=0$. Then (27) shows that $w$ can have at most simple zeros, and $m(r, 1 / w) \leq S(r, V) \leq S(r, F) \leq S(r, f)$, using (23). This gives

$$
m\left(r, \frac{1}{f}\right) \leq m\left(r, \frac{w}{f}\right)+m\left(r, \frac{1}{w}\right)=S(r, f)
$$

and

$$
T(r, f) \leq N\left(r, \frac{1}{f}\right)+S(r, f) .
$$

Finally, writing $1 / f=(w / f)(1 / w)$ and noting again that each $f^{(j)} / f$ has poles of multiplicity at most $j$, we get

$$
T(r, f) \leq N\left(r, \frac{1}{f}\right)+S(r, f) \leq N^{(k)}\left(r, \frac{1}{f}\right)+S(r, f),
$$

which is a stronger inequality than (7).

Assume henceforth that $H$ is meromorphic in the plane. A straightforward integration from (23), using (24) and Lemma 4.1, gives a polynomial $P$ of degree at most $k-1$ such that

$$
f=\frac{H^{k+1}+H P(H)+(-1)^{k+1}}{k ! H\left(H^{\prime}\right)^{k}}=\frac{\prod_{j=1}^{k+1}\left(H-c_{j}\right)}{k ! H\left(H^{\prime}\right)^{k}},
$$


with constant roots $c_{j}$. If all of the $c_{j}$ are equal, then $f$ obviously has the form (9). We assume next, with no loss of generality, that $c_{1} \neq c_{2}$. Then (29) gives

$$
\frac{1}{k ! f}=\frac{H^{\prime} H}{\left(H-c_{1}\right)\left(H-c_{2}\right)} \prod_{3 \leq j \leq k+1}\left(\frac{H^{\prime}}{H-c_{j}}\right)
$$

and so

$$
\frac{1}{f}=\left(\frac{d_{1} H^{\prime}}{H-c_{1}}+\frac{d_{2} H^{\prime}}{H-c_{2}}\right) \prod_{3 \leq j \leq k+1}\left(\frac{H^{\prime}}{H-c_{j}}\right),
$$

with $d_{1}, d_{2}$ constants. But (23) and (30) give

$$
m\left(r, \frac{1}{f}\right) \leq S(r, H) \leq S(r, F) \leq S(r, f) .
$$

Since (30) implies that $f$ cannot have a zero of multiplicity greater than $k$, we obtain (28) again.

Finally, suppose that $a_{k-1} \equiv 0$, and that (8) and (9) hold. Then $H$ is a linear function and $f$ is a rational function, with $f(\infty)=\infty$. Thus

$$
T(r, f)=N\left(r, \frac{1}{f}\right)+O(1)
$$

for large $r$, and $f$ has just one zero, of multiplicity $k+1$, so that (7) still holds.

\section{Proof of Theorems 1.4, 1.5 and 1.6}

First, let $k, M_{k}, N_{k}$ be as in the hypotheses of Theorem 1.5 and suppose that $f \in \mathfrak{F}\left(k, M_{k}, N_{k}, \mathbb{C}\right)$ is non-constant. Then $f^{(k)}$ is non-constant, since $M_{k}>k$, and so $f$ satisfies the hypotheses of Theorem 1.7, with $L_{k}=D^{k}$. But

$$
\bar{N}\left(r, \frac{1}{f}\right) \leq M_{k}^{-1} T(r, f)+O(1), \quad \bar{N}\left(r, \frac{1}{f^{(k)}-1}\right) \leq N_{k}^{-1} T\left(r, f^{(k)}\right)+O(1),
$$

and a contradiction arises from (7), since $T\left(r, f^{(k)}\right) \leq(k+1) T(r, f)+S(r, f)$.

To establish Theorem 1.6, which will obviously in turn prove the normality criterion of Theorem 1.5, we apply the rescaling lemma referred to in the introduction. This result, in the present form due to Chen and Gu [3], extends earlier work of Zalcman [21] and Pang [13, 14]. For a survey of these results and their applications, see [22].

LEMMA 5.1. Let $\alpha>0$ and let $H$ be a family of functions meromorphic in the plane domain $D$ such that for every $h$ in $H$ all zeros of $h$ in $D$ have multiplicity greater than $\alpha$. If $H$ is not normal at $z_{0} \in D$ then there exist sequences $z_{n} \rightarrow z_{0}, h_{n} \in H, \rho_{n} \rightarrow 0$, such that, as $n \rightarrow \infty$, the functions $\rho_{n}^{-\alpha} h_{n}\left(z_{n}+\rho_{n} z\right)$ converge locally spherically uniformly in $\mathbb{C}$ to a non-constant meromorphic function $h$ of finite order. 
Assume now that $k, D, a_{j}, \mathfrak{G}$ satisfy the hypotheses of Theorem 1.6, but that $\mathfrak{G}$ is not normal. Since normality is a local property we may further assume that $0 \in D$ and that $\mathfrak{G}$ is not normal at 0 , and that the $a_{j}$ are bounded on $D$. Since $M_{k}>k$ we may apply Lemma 5.1, which gives sequences $z_{n} \rightarrow 0, f_{n} \in \mathfrak{G}, \rho_{n} \rightarrow 0$, such that the functions $g_{n}(z)=\rho_{n}^{-k} f_{n}\left(z_{n}+\rho_{n} z\right)$ converge locally spherically uniformly in the plane to a non-constant meromorphic function $g$.

A standard application of Hurwitz' theorem shows that all zeros of $g$ have multiplicity at least $M_{k}$. Next, let $\zeta$ be a zero of $g^{(k)}-1$. Then $g$ is bounded near $\zeta$ and $g_{n}$ converge uniformly to $g$ on a neighbourhood $U$ of $\zeta$, with respect to the standard metric. With $F_{n}=L\left(f_{n}\right)$ this gives

$$
F_{n}\left(z_{n}+\rho_{n} z\right)=g_{n}^{(k)}(z)+\sum_{j=0}^{k-1} \rho_{n}^{k-j} a_{j}\left(z_{n}+\rho_{n} z\right) g_{n}^{(j)}(z) \rightarrow g^{(k)}(z)
$$

locally uniformly on $U$. Applying Hurwitz' theorem again we deduce that all zeros of $g^{(k)}-1$ have multiplicity at least $N_{k}$, and this contradicts the first part of Theorem 1.5.

We turn now to the proof of Theorem 1.4. The obvious examples

$$
f_{n}(z)=2 n(z-a)^{k}, \quad n \in \mathbb{N}, a \in \mathbb{C},
$$

show that there is nothing to prove if $M \leq k$, since $f_{n} \in \mathfrak{F}(k, k, \infty, \mathbb{C})$ and there is no neighbourhood of $a$ on which the $f_{n}$ form a normal family. On the other hand, if $M>k$ then the same application of Lemma 5.1 as in the proof of Theorem 1.6 shows that if $\mathfrak{F}(k, M, N, D)$ is not normal then $\mathfrak{F}(k, M, N, \mathbb{C})$ has non-constant elements, while if $f \in \mathfrak{F}(k, M, N, \mathbb{C})$ is non-constant, then the functions $h_{n}(z)=n^{-k} f(n z), n \in \mathbb{N}$, form a family which is not normal at 0 . Obviously Theorem 1.4, together with the first part of Theorem 1.5, gives an alternative way to prove the normality criterion of Theorem 1.5.

\section{Proof of Theorem 1.8}

We first prove that there exists a positive integer $T_{k}$ with the property that $\mathfrak{F}(k, k+$ $\left.2, T_{k}, \mathbb{C}\right)$ contains only constant functions. Assuming that no such $T_{k}$ exists, we obtain for each large positive integer $n$ a non-constant function $f_{n} \in \mathfrak{F}(k, k+2, n, \mathbb{C})$, and there is no loss of generality in assuming that $\left|f_{n}(0)\right| \leq 1$ for each $n$. Applying Milloux' inequality (13) to $f_{n}$ gives

$$
T\left(r, f_{n}\right)<\bar{N}\left(r, f_{n}\right)+k \bar{N}\left(r, \frac{1}{f_{n}}\right)+\bar{N}\left(r, \frac{1}{f_{n}^{(k)}-1}\right)+S\left(r, f_{n}\right),
$$


and so each $f_{n}$ has at least one pole. Replacing $f_{n}(z)$ if necessary by $R_{n}^{-k} f_{n}\left(R_{n} z\right)$ for some large positive $R_{n}$, we may therefore assume that the family $f_{n}$ is not equicontinuous, and hence not normal, at 0 . Applying Lemma 5.1 we obtain sequences $n_{q} \rightarrow \infty, z_{q} \rightarrow 0, \rho_{q} \rightarrow 0$, such that as $q \rightarrow \infty$ the functions $\rho_{q}^{-k} f_{n_{q}}\left(z_{q}+\rho_{q} z\right)$ converge locally spherically uniformly in the plane to a non-constant meromorphic function $g$ of finite order. As in the proof of Theorem 1.5 we deduce that all zeros of $g$ have multiplicity at least $k+2$, and that $g^{(k)}-1$ has no zeros. This contradicts Propositions 1.9 and 1.10 .

Applying Lemma 5.1 in the same way to $\mathfrak{F}\left(k, k+2, T_{k}, D\right)$ we now see that $\mathfrak{F}\left(k, k+2, T_{k}, D\right)$ is normal for every plane domain $D$.

\section{Proof of Theorem 1.11}

LEMMA 7.1. Let $k \geq 2$ and let $f$ be meromorphic in the plane such that $f^{(k)}$ is non-constant and satisfies

$$
m\left(r, f^{(k)}\right)=S\left(r, f^{(k)}\right), \quad N(r, f) \leq N_{1}(r, f)+S\left(r, f^{(k)}\right),
$$

in which $N_{1}(r, f)$ counts the simple poles of $f$. Assume further that, for some $N \geq 2$,

$$
\bar{N}\left(r, \frac{1}{f^{(k)}-1}\right) \leq \frac{1}{N} T\left(r, f^{(k)}\right)+S\left(r, f^{(k)}\right) .
$$

Then either

$$
f^{(k)}(z)=1-\frac{1}{(\alpha z+\beta)^{k+1}} \quad, \quad \alpha, \beta \in \mathbb{C}, \quad \alpha \neq 0
$$

or

$$
N(k-1) \leq 2(k+1) \text {. }
$$

Proof. Assume that $f$ is as in the hypotheses. By (31) we have

$$
T\left(r, f^{(k)}\right) \leq(k+1) N(r, f)+S\left(r, f^{(k)}\right) .
$$

Define $M$ by (20), with $F=f^{(k)}$ and $a_{k-1}=0$. Then $M$ has a zero of multiplicity at least $k$ at each simple pole of $f$, using Lemma 2.2.

If $M \equiv 0$ then we obtain (33). Assume henceforth that $M \not \equiv 0$. Then we have, using (20), the lemma of the logarithmic derivative and (31),

$$
\begin{aligned}
k N(r, f) & \leq N(r, 1 / M)+S\left(r, f^{(k)}\right) \leq T(r, M)+S\left(r, f^{(k)}\right) \\
& \leq N(r, M)+S\left(r, f^{(k)}\right) \\
& \leq \bar{N}\left(r, \frac{1}{f^{(k)}-1}\right)+\bar{N}\left(r, \frac{f^{(k)}-1}{f^{(k+1)}}\right)+S\left(r, f^{(k)}\right)
\end{aligned}
$$




$$
\begin{aligned}
& \leq \bar{N}\left(r, \frac{1}{f^{(k)}-1}\right)+\bar{N}\left(r, \frac{f^{(k+1)}}{f^{(k)}-1}\right)+S\left(r, f^{(k)}\right) \\
& \leq 2 \bar{N}\left(r, \frac{1}{f^{(k)}-1}\right)+N(r, f)+S\left(r, f^{(k)}\right),
\end{aligned}
$$

which leads to, using (32) and (35),

$$
(k-1) N(r, f) \leq \frac{2}{N} T\left(r, f^{(k)}\right)+S\left(r, f^{(k)}\right) \leq\left(\frac{2(k+1)}{N}+o(1)\right) N(r, f)
$$

outside a set of finite measure, from which (34) follows at once.

Assume now that $f$ satisfies the hypotheses of Theorem 1.11. We write

$$
f(z)=\frac{c U(z)}{V(z)}, \quad c \in \mathbb{C} \backslash\{0\}, \quad U(z)=\prod_{j=1}^{s}\left(z-a_{j}\right)^{m_{j}}, \quad V(z)=\prod_{j=1}^{t}\left(z-b_{j}\right)^{n_{j}},
$$

where the $a_{j}$ and $b_{j}$ are distinct. By hypothesis we have $m_{j} \geq M$ for all $j$. Moreover, $u=\operatorname{deg} U=\sum_{j=1}^{s} m_{j}$ and $v=\operatorname{deg} V=\sum_{j=1}^{t} n_{j}$. We put $l=k-1$ and $h=f^{(l)}$. Obviously if $k=1$ then $h=f$, and in the general case we can write

$$
h(z)=\frac{P(z)}{Q(z)}, \quad P(z)=R(z) \prod_{j=1}^{s}\left(z-a_{j}\right)^{m_{j}-l}, \quad Q(z)=\prod_{j=1}^{t}\left(z-b_{j}\right)^{n_{j}+l},
$$

in which $R$ is a polynomial.

LEMMA 7.2. Let. $p=\operatorname{deg} P$ and $q=\operatorname{deg} Q$. Then we have $q=v+$ lt. Further, $p=u+l(t-1)$ if $u \geq v+l$ or $u<v$, and $p \leq v+l(t-1)-1$ otherwise.

Proof. We have $f(z) \sim c z^{u-v}$ as $z \rightarrow \infty$. If $u \geq v+l$ or $u<v$ we obtain $h(z) \sim c^{*} z^{u-v-l}$ as $z \rightarrow \infty$, with $c^{*}=c(u-v)(u-v-1) \cdots(u-v-l+1) \neq 0$. If $v \leq u<v+l$ we have instead $h(z)=O\left(|z|^{-l-1}\right)$ as $z \rightarrow \infty$. Hence we get $p-q=u-v-l$ if $u \geq v+l$ or $u<v$, and $p-q \leq-l-1$ otherwise.

We now define $g(z)=z-h(z)$ and $d=\operatorname{deg} g$. If $f$ is a polynomial, then $\operatorname{deg} f \geq M \geq k+1$. Thus $d=\operatorname{deg} h=\operatorname{deg} f-l \geq 2$ in this case. If $f$ has a pole and $k \geq 2$, then $h$ has a multiple pole and again we find that $d \geq 2$. Finally if $k=1$ and $f$ has at least one pole then either $d \geq 2$ or $f$ has the form (10). We may therefore assume henceforth that $d \geq 2$, so that $g$ is not constant and not a Möbius transformation, and the Fatou-Julia iteration theory can be applied to $g$.

The zeros $a_{j}$ of $f$ are then fixed points of $g$ of multiplier 1 , each with $m_{j}-$ $l-1=m_{j}-k$ attached parabolic basins [16, page 75]. Altogether there are thus $\sum_{j=1}^{s}\left(m_{j}-k\right)=u-s k$ parabolic basins of $g$ attached to the zeros of $f$. If $p \leq q$, then $\infty$ is also a fixed point of multiplier 1 , with $q-p+1$ parabolic basins attached to it. This gives: 
LEMMA 7.3. With $w=\max \{q-p+1,0\}$, the function $g$ has $L \geq u-s k+w$ parabolic basins.

Since $u=\sum_{j=1}^{s} m_{j} \geq M s$ we have $s \leq u / M$ and thus $u-s k \geq(1-k / M) u$. Thus using Lemma 7.3 we get

$$
L \geq u-s k+w \geq\left(1-\frac{k}{M}\right) u+w .
$$

We now compute the number $C$ of zeros of $g^{\prime}$, with multiplicities counted. To do so we note that the zeros of $g^{\prime}$ are critical points of $g$ and that $g$ has $2 d-2$ critical points. Besides the zeros of $g^{\prime}$, the only critical points are multiple poles and possibly the point at $\infty$. The contribution from the poles is given by

$$
\sum_{j=1}^{t}\left(n_{j}+l-1\right)=v+t(l-1) .
$$

If $\infty$ is a critical point, then we denote by $r$ its order, and we put $r=0$ otherwise. Then

$$
C=2 d-2-v-t(l-1)-r \leq 2 d-2-v-t(l-1) .
$$

We shall see below that $d \in\{p, q, q+1\}$, but we first note that if $d=q+1$ then $d=v+t l+1$ and

$$
2 d-2-v-t(l-1)=v+t(l+1)=v+k t \leq(k+1) v .
$$

Hence if $d=q+1$ we obtain

$$
C \leq(k+1) v-r \leq(k+1) v .
$$

If $d=q$ the same computation yields

$$
C \leq(k+1) v-2-r \leq(k+1) v-2 .
$$

Further, we have strict inequality in (38) and (39) unless $v=t$, that is, all poles of $f$ are simple.

We now distinguish two cases, depending on whether or not $g$ has a pole at $\infty$.

\section{Case $1 g(\infty)=\infty$.}

Then each parabolic basin of $g$ contains a finite critical point of $g$ which is not a pole. These critical points are zeros of $g^{\prime}$ and thus, by hypothesis, have multiplicity at least $N$. This gives

$$
N L \leq C
$$

This case now requires four subcases, depending on the relative sizes of $u$ and $v$. 
Subcase (i) $u<v$.

Then Lemma 7.2 gives $p=u+(t-1) l<q=v+t l$, and we have $d=q+1$ and

$$
w=q-p+1=v-u+l+1=v-u+k .
$$

Combining this with (36), (38) and (40) we obtain

$$
N\left(1-\frac{k}{M}\right) u+N(v-u+k) \leq(k+1) v .
$$

This can be rewritten as

$$
-\frac{k}{M} u+\left(1-\frac{k+1}{N}\right) v \leq-k<0 .
$$

Since $u<v$ this implies that

$$
\left(1-\frac{k}{M}-\frac{k+1}{N}\right) v<-k<0,
$$

and (11) follows.

Subcase (ii) $v \leq u \leq v+l-1$.

This time Lemma 7.2 gives $p \leq v+l(t-1)-1=q-l-1 \leq q$, and we have $d=q+1$, and $w=q-p+1 \geq l+2$. Now (36), (38) and (40) yield

$$
N\left(1-\frac{k}{M}\right) u+N(l+2) \leq(k+1) v
$$

and thus

$$
\left(1-\frac{k}{M}\right) u-\frac{k+1}{N} v \leq-l-2<0 .
$$

Since $v \leq u$ this implies that

$$
\left(1-\frac{k}{M}-\frac{k+1}{N}\right) u \leq-l-2<0
$$

and (11) follows.

Subcase (iii) $u=v+l$.

Then $p=u+(t-1) l=v+t l=q, d=q+1$, and $w=1$. In a manner similar to that previously we deduce from (36), (38) and (40) that

$$
N\left(1-\frac{k}{M}\right) u+N \leq(k+1) v=(k+1) u-(k+1) l
$$

and thus

$$
\left(1-\frac{k}{M}-\frac{k+1}{N}\right) u \leq-1-\frac{(k+1) l}{N}<0 .
$$

Again (11) follows. 
Subcase (iv) $u \geq v+l+1$.

Then $p=u+(t-1) l>v+t l=q, d=p$, and $w=0$. If $p>q+1$, then $\infty$ is a critical point of order $p-q-1$. Thus we can take $r=p-q-1$ in (37) and obtain

$$
\begin{aligned}
C & \leq 2 p-2-v-t(l-1)-p+q+1=p+q-v-t(l-1)-1 \\
& =u+(t-1) l+t l-t(l-1)-1=u+k t-k \leq u+k v-k \\
& \leq u+k(u-k)-k=(k+1) u-k^{2}-k .
\end{aligned}
$$

Combining this with (36) and (40) we obtain $N(1-k / M) u \leq(k+1) u-k^{2}-k$. This yields $(1-k / M-(k+1) / N) u \leq-\left(k^{2}+k\right) / N<0$ and hence (11).

Case $2 g(\infty) \neq \infty$.

Then $p=q+1, d=q, w=0$, and $u=v+l+1=v+k$.

Now each of the parabolic basins of $g$ attached to the $a_{j}$, with at most one exception, contains a finite critical point of $g$ which is not a pole of $g$, and hence is a zero of $g^{\prime}$. Instead of (40) we thus only obtain

$$
N(L-1) \leq C .
$$

Since $g(z)=g(\infty)+O\left(z^{-l-1}\right)$ we see that $\infty$ is a critical point of $g$, of order at least $l$. Thus $r \geq l$ in (39). We hence deduce from (36), (39) and (41) that

$$
N\left(1-\frac{k}{M}\right) u-N \leq(k+1) v-2-l .
$$

We observe further that if $k=1$ then (42) is a strict inequality. For if $k=1$ and $g$ has a critical point at $\infty$ then we may take $r>l=0$ in (39), while if $\infty$ is not a critical point we have (40) again.

Since $l=k-1$ and $v=u-k$, (42) yields

$$
N\left(1-\frac{k}{M}\right) u-N \leq(k+1) u-(k+1)^{2}
$$

and thus

$$
\left(1-\frac{k}{M}-\frac{k+1}{N}\right) u \leq 1-\frac{(k+1)^{2}}{N},
$$

again with strict inequality if $k=1$. If $N<(k+1)^{2}$, then the right hand side of (43) is negative and (11) follows.

It remains only to dispose of the subcase $N \geq(k+1)^{2}$. Suppose that $N>(k+1)^{2}$ but that (11) does not hold. If $k / M+(k+1) / N=1$, then

$$
\frac{k}{M}=1-\frac{k+1}{N}>1-\frac{1}{k+1}=\frac{k}{k+1}
$$


and thus $M<k+1$, contradicting the hypotheses. If $k / M+(k+1) / N<1$, then the right hand side of (43) is positive and we obtain

$$
u \leq \frac{1-(k+1)^{2} / N}{1-k / M-(k+1) / N}=k+1-\frac{k(M-k-1)}{M(1-k / M-(k+1) / N)} \leq k+1 .
$$

Thus $u=k+1$ and $v=1$. This implies, recalling that $g(\infty)$ is finite, that $f$ has the form (10).

Finally suppose that $N=(k+1)^{2}$. Then (43) yields

$$
1-\frac{k}{M}-\frac{k+1}{N} \leq 0
$$

with strict inequality if $k=1$. Assuming that (11) fails, we must therefore have $k \geq 2$ and equality in (44), which forces $M=k+1$. Further, we must have equality in (43) and in all inequalities leading to (43), in particular in (39), so that all poles of $f$ must be simple. Since $g(\infty)$ is finite we have $f^{(k)}(\infty)=1$, and applying Lemma 7.1 we see that $f^{(k)}$ must satisfy (33). Integrating $k$ times and using the fact that $M=k+1$ we get (10) again.

\section{Acknowledgements}

We thank Yuefei Wang and Yang Le for helpful comments. We also thank the referee for a careful reading of the manuscript and some valuable suggestions.

Part of this work was done while the first author was visiting the U.K. He thanks Walter Hayman for arranging this visit, and the Royal Society and the London Mathematical Society for support. The first author is also supported by the German-Israeli Foundation for Scientific Research and Development, G.I.F., G -643-117.6/1999 and INTAS-99-00089. The second author thanks the DAAD for supporting a visit to Kiel in June-July 2002.

\section{References}

[1] W. Bergweiler, 'Normality and exceptional values of derivatives', Proc. Amer. Math. Soc. 129 (2001), 121-129.

[2] W. Bergweiler and A. Eremenko, 'On the singularities of the inverse to a meromorphic function of finite order', Rev. Mat. Iberoamericana 11 (1995), 355-373.

[3] Chen Huai Hui and Gu Yong Xing, 'Improvement of Marty's criterion and its application', Sci. China Ser. A 36 (1993), 674-681.

[4] Chen Zhen Hua, 'Normality of families of meromorphic functions with multiple valued derivatives', Acta Math. Sinica 30 (1987), 97-105. 
[5] Chi Tai Chuang and L. Yang, 'Distributions of the values of meromorphic functions', in: Analytic functions of one complex variable, Contemp. Math. 48 (Amer. Math. Soc., Providence, RI, 1985) pp. $21-63$.

[6] A. Eremenko, 'Distribution of zeros of some real polynomials and iteration theory', preprint, (Institute for Low Temperature Physics and Engineering, Kharkov, 1989).

[7] Gu Yong Xing, 'A criterion for normality of families of meromorphic functions', Sci. Sinica Special Issue on Math. 1 (1979), 267-274.

[8] W. K. Hayman, 'Picard values of meromorphic functions and their derivatives', Ann. of Math. (2) 70 (1959), 9-42.

[9] - Meromorphic functions (Clarendon Press, Oxford, 1964).

[10] M. Heckner, 'A remark on Langley's generalisation of Hayman's alternative', Result. Math. 25 (1994), 54-59.

[11] J. K. Langley, 'On Hayman's alternative', Mathematika 32 (1985), 139-146.

[12] _ 'A lower bound for the number of zeros of a meromorphic function and its second derivative', Proc. Edinburgh Math. Soc. 39 (1996), 171-185.

[13] Pang Xuecheng, 'Bloch's principle and normal criterion', Sci. China Ser. A 32 (1989), 782-791.

[14] - 'On normal criterion of meromorphic functions', Sci. China Ser. A 33 (1990), 521-527.

[15] W. Schwick, 'Normality criteria for families of meromorphic functions', J. Analyse Math. 52 (1989), 241-289.

[16] N. Steinmetz, Rational iteration, de Gruyter Studies in Mathematics 16 (Walter de Gruyter, Berlin, 1993).

[17] Y. F. Wang and M. Fang, 'Picard values and normal families of meromorphic functions with multiple zeros', Acta Math. Sinica New Ser. 14 (1998), 17-26.

[18] L. Yang, 'Precise fundamental inequalities and sums of deficiencies', Sci. China Ser. A 34 (1991), 157-165.

[19] L. Yang and G. H. Zhang, 'Recherches sur la normalité des familles de fonctions analytiques à des valeurs multiples. I. Un nouveau critère et quelques applications', Sci. Sinica 14 (1965), $1258-1271$.

[20] - ' Recherches sur la normalité des familles de fonctions analytiques à des valeurs multiples. II. Généralisations', Sci. Sinica 15 (1966), 433-453.

[21] L. Zalcman, 'A heuristic principle in complex function theory', Amer. Math. Monthly 82 (1975), 813-817.

[22] _ _ 'Normal families: new perspectives', Bull. Amer. Math. Soc. (N.S.) 35 (1998), 215-230.

Mathematisches Seminar

Christian-Albrechts-Universität zu Kiel

Ludewig-Meyn Str. 4

D-24098 Kiel

Jermany

¿-mail: bergweiler@math.uni-kiel.de
School of Mathematical Sciences

University of Nottingham

NG7 2RD

UK

e-mail: jkl@maths.nott.ac.uk 
